\title{
Cell free DNA: A Novel Predictor of Neurological Outcome after Intravenous Thrombolysis and/or Mechanical Thrombectomy in Acute Ischemic Stroke Patients
}

\author{
Atulabh Vajpeyee, MD, DM, FINS', Teddy Wijatmiko, MD', \\ Manisha Vajpeyee, PhD', Onjal Taywade, MD²
}

Purpose: Several blood markers have been evaluated in stroke patients, but their role remains limited in clinical practice. This study was designed to evaluate the utility of cell free DNA (cf DNA) in stroke patients undergoing therapeutic intervention in the form of mechanical thrombectomy in acute ischemic stroke patients.

Materials and Methods: Twenty-six patients with ischemic stroke who were managed with interventions like intravenous thrombolysis (IVT) and mechanical thrombectomy were recruited consecutively in this study. The cf DNA was extracted by using circulating nucleic acid kit and measured by real-time quantitative PCR assay for $\beta$-globin gene. The neurological outcome was measured by modified Rankin scale (mRS) score at three months after the onset of symptoms.

Results: Cf DNA levels correlated with severity of stroke at the time of admission $(r=0.421, P=0.032)$ and poor outcome at three months $(r=0.606, P=0.001)$. Therapeutic intervention in the form of mechanical thrombectomy or IVT was associated with improved outcome in patients with of DNA $<10,000$ kilogenome-equivalents/L $(P=<0.05)$.

Conclusion: Cf DNA level correlated well with the 3 month outcome in acute ischemic stroke patients. It can be a potential supplementary marker to predict neurological outcome after therapeutic intervention.

Key Words : Cell free DNA; Stroke; Mechanical thrombectomy; Intravenous thrombolysis

Stroke has become a major cause of death and disability across all countries. ${ }^{1}$ Unlike myocardial infarction, there are no established markers for assess- ment of disease severity or predicting outcome in acute stroke. Various studies have evaluated biomarkers to distinguish ischemic stroke from stroke mimics,

'Deparments of Neurology and 'Biochemistry, Pacific Medical College \& Hospital, Pacific Medical University, Udaipur, India Received August 3, 2017; Revised January 27, 2018; Accepted Feburary 7, 2018

Correspondence to: Atulabh Vajpeyee, MD

Pacific Medical College \& Hospital, Pacific Medical University, Bhilo Ka Bedla, Udaipur, Rajasthan, India. Pin:-313001

Tel. +92.294.392.0000 (Ext.-2271) Fax.+92.294.392.0081 E-mail: researchudr@gmail.com

This is an Open Access article distributed under the terms of the Creative Commons Attribution Non-Commercial License (http://creativecommons.org/licenses/by-nc/3.0) which permits unrestricted non-commercial use, distribution, and reproduction in any medium, provided the original work is properly cited. 
determine etiology, predict severity and outcomes including early neurological deterioration and hemorrhagic complications. Stroke biomarkers can also identify patients who may benefit from specific therapies, including recanalization strategies using intravenous thrombolysis, and decompressive hemicraniectomy.

To date, a number of biomarkers have been identified to answer focused clinical questions. There are CNS tissue injury biomarkers like S100B, GFAP, NSE, NMDA-R Ab, MBP; inflammatory biomarkers like HsCRP, IL-6, TNF- $\alpha$, VCAM 1, ICAM 1, MMP2, MMP9, Lp-PLA2; coagulation biomarkers like ApoCI, ApoC-III, fibrinogen, D-Dimer, vWF and other biomarkers like plasma DNA, PARK7, NDKA B-type neurotrophic growth factor. None of these has acquired sufficient sensitivity and specificity as seen for biomarkers for coronary artery disease. ${ }^{2}$

Cell-free DNA (cf DNA) refers to the double helix DNA fragments released from nucleated cells and circulating freely in blood stream., ${ }^{3,4}$ As both ischemic and hemorrhagic stroke involve cell death and disruption of the blood-brain barrier, it is hypothesized that $\mathrm{cf}$ DNA would be liberated into the plasma soon after the onset of stroke, and hence, may be useful for assessing disease severity and prognosis. Unlike myocardial infarction, the brain has the blood brain barrier limiting the use of biomarkers. Also, the heart does not usually have hemorrhagic complications like those seen in the brain with recanalization or thrombolytic drugs. We still need to have readily available, simple, and costeffective blood biomarker to predict not only stroke outcome, but also utility or futility of revascularization procedures like intravenous thrombolysis (IVT) and mechanical thrombectomy.

Long term care and rehabilitation is a huge financial burden for the families of the stroke survivors. With the advancement of modern treatment modalities and the availability of stroke care units, the number of stroke survivors is rising. There is a need for the evaluation of blood biomarkers to predict the neurological outcome in such patients for optimal utilization of resources. To the best of our knowledge, this is the first study which hypothesizes that cf DNA can be used as a marker for monitoring disease progress and assessing post intervention outcome in stroke patients.

\section{MATERIALS AND METHODS}

\section{Patients and endovascular therapy}

Twenty-six patients with proximal middle cerebral artery occlusion who underwent therapeutic intervention at our institute were recruited consecutively into the study. Venous blood sample for cf DNA was collected within first 6 hours of stroke onset. Leptomeningeal collateral status was evaluated and poor collateral status was defined as $\leq 50 \%$ collateral filling of the perfusion territory of the affected middle cerebral artery (MCA) territory. ${ }^{5}$

Four groups of researchers responsible for clinical data collection, therapeutic intervention, assessment of clinical outcome, and DNA analysis were blinded to one another. Relevant medical data, including time of onset of symptoms, history of hypertension, diabetes mellitus, ischemic heart disease, hyperlipidemia, smoking, alcohol and medication were recorded. All the patients received standard clinical, laboratory and imaging workup, including noncontrast brain computed tomography (NCCT) and/or magnetic resonance imaging (MRI) with complete angiogram (CTA and or MRA) to determine the exact nature of the stroke. The Alberta stroke programm early CT score (ASPECTS) was measured on the baseline NCCT scans. We repeated the NCCT brain immediately after the IVT and mechanical thrombectomy procedure and further repeated NCCT on the 7th day or in between if needed.

The National Institutes of Health Stroke Scale (NIHSS) assessed the severity of stroke clinically at the time of admission. ${ }^{6}$ Modified Rankin scale (mRS) was used to determine the clinical outcome. ${ }^{7}$ The primary outcome measure was post-stroke modified Rankin scale score at three months after the onset of symptoms. MRS 0-2 was considered as good outcome, whereas $\mathrm{mRS} \geq 3$ was considered as poor outcome.

If the patient presented within 4.5 hours after onset of stroke, IV tissue plasminogen activator ( $t-P A)$ (0.9 $\mathrm{mg} / \mathrm{kg}$ ) was started as per NINDS criteria. ${ }^{8}$ If proximal large vessel occlusion was seen, we used the Solitaire ${ }^{\mathrm{TM}}$ FR device for the interventional procedure, the size of which was selected per device-specific instructions for use. IV sedation or general anesthesia may be administered to assure subject comfort and safety, if required. Procedures were performed using biplane angiography system (Allura xper FD 20-15, Phillips). Several retrieval passes with the Solitaire revascularization devices were performed, up to a maximum of three retrievals in the same vessel, and a maximum of two retrievals per device. The microcatheter was positioned across the visible clot, and the stent retrieval device was deployed at the desired location by pulling back the microcatheter. Resheathing of the Solitaire was done, if required, but not more than twice. Thrombus retrieval 


\section{Cf DNA- Outcome Prediction after Mechanical Thrombectomy in Stroke}

was performed under negative aspiration (20 cc Syringe. The final angiogram was done to evaluate the restoration of flow (TICI flow). ${ }^{9}$ The median door-toneedle time was 30 minutes for IVT, and groin puncture-to-recanalization time was thirty minutes. Time from door to recanalization was 90 minutes.

\section{Extraction of cf DNA and real-time PCR}

Five $\mathrm{mL}$ venous blood sample upon arrival in the emergency room was collected in an EDTA tube and centrifuged at $14,000 \mathrm{~g}$ for $20 \mathrm{~min}$. Supernatant plasma was separated and stored at $-80^{\circ} \mathrm{C}$ till further processing. The cf DNA was extracted from $1 \mathrm{ml}$ plasma with QIAamp ${ }^{\circledR}$ Circulating Nucleic Acid Kit (Qiagen, Germany) as recommended by the manufacturer. The theoretical and practical aspects of real-time quantitative PCR are already described in detail in the literature. ${ }^{10,11}$ The cf DNA extraction took 40 minutes.

The cf DNA estimation was done by a real-time quantitative PCR assay for $\beta$-globin gene (QuantiNova probe RT PCR kit- Qiagen) on Rotor-Gene Q platform (Qiagen, Germany). The $\beta$-globin gene PCR system consists of the amplification primers beta-globin-354F (5' -GTG CAC CTG ACT CCT GAG GAG A-3' $)$ and beta-globin-455R (5' -CCT TGA TAC CAA CCT GCC CAG-3' ) and a dual-labeled fluorescent PCR probe beta-globin-402T [5' -(VIC $\left.{ }^{\circledR}\right)$ AAG GTG AAC GTG GAT GAA GTT GGT GG-3' $].{ }^{11}$ When applied to serial dilutions of human genomic DNA, this real-time quantitative $\beta$-globin PCR assay was able to detect the DNA equivalent from a single cell. The imprecision of this system has been reported previously, with a $\mathrm{CV}$ of the threshold cycle of $1.1 \%$. The quantitative results from real time PCR were expressed as cf DNA kilogenome equivalents/L. ${ }^{3,410}$ The mean normal cut off value from healthy individuals is 3,154 kilogenome equivalents/L. ${ }^{4}$

\section{Statistical analysis}

Descriptive statistics, data comparison tests $\left(\boldsymbol{x}^{2}\right.$, Fisher exact, Mann-Whitney and Kruskal-Wallis tests), correlations (Spearman rank tests) were carried out using Statistical Package for Social Sciences (SPSS, Chicago, IL, USA). P value $<0.05$ was considered significant. Various variables used were age, sex, duration of sample collection from time of stroke onset, risk factors like diabetes, hypertension, hyperlipidemia, history of ischemic heart disease, tobacco use, smoking, clinical criteria like NIHSS score at admission, mRS score at three months, imaging criteria for core infarct size by ASPECT CT score and CTA or
MRA for proximal M1 occlusion, blood parameters like cell free DNA, blood glucose, lipid profile and HsCRP.

\section{RESULTS}

All patients enrolled had proximal M1 MCA occlusion with similar risk factor distribution, and intervention following standard guidelines for IVT with t-PA and mechanical thrombectomy. Most of these patients ( 20 out of 26 patients) had $\leq 50 \%$ collateral filling of the perfusion territory of the affected MCA territory. TICI $2 \mathrm{~b}$ and TICI 3 flow was achieved in all patients $(n=15)$ who underwent mechanical thrombectomy. No association was found between cf DNA level and age, gender or duration of blood sample collection from onset of stroke as well as other stroke risk factors (Table 1). There was moderate correlation between the cf DNA level and NIHSS at admission $(r=0.421$, $\mathrm{P}=0.032$ ) while strong correlation with $\mathrm{mRS}$ score at

Table 1. Characteristics of Stroke Subjects and Comparison with Cell Free DNA

\begin{tabular}{lcc}
\hline Characteristics & Results $(\mathrm{n}=26)$ & P-value \\
\hline Age (years) Mean (Range) & $62.88(40-80)$ & 0.378 \\
Gender-Male (\%) & $15(57.7 \%)$ & 0.949 \\
\hline Risk factors & No. of patients (\%) & P-value \\
\hline Hypertension & $13(50 \%)$ & 0.102 \\
Diabetes mellitus & $5(19.2 \%)$ & 0.408 \\
Ischemic heart disease & $3(11.3 \%)$ & 0.887 \\
Hyperlipidemia & $9(34.6 \%)$ & 0.236 \\
Duration in hours\# (range) & $5.48 \pm 2.27$ & 0.911 \\
\hline Clinical assessment scale scores & Mean \pm SD (range) \\
\hline NIHSS admission & $15.73 \pm 8.92$ \\
mRS admission & $4.12 \pm 1.24$ \\
mRS 1 month & $2.92 \pm 1.78$ \\
mRS 3 month & $2.77 \pm 1.81$ \\
ASPECTS & $7.04 \pm 2.28$
\end{tabular}

Therapeutic Intervention Thrombectomy plus IV thrombolysis $(n=26)$

\begin{tabular}{lc} 
Mechanical Thrombectomy & $15(57.69 \%)$ \\
IV Thrombolysis & $16(61.53 \%)$ \\
\hline Cf DNA median* (range) & $8784(902-33138)$ \\
\hline
\end{tabular}

* kilogenome equivalents/L 
three months $(\mathrm{r}=0.606, \mathrm{P}=0.001)$. The cf DNA concentration of patients with poor outcome (mRS score $\geq 3$ ) was significantly higher than that of patients with good outcome $(\mathrm{P}<0.001)$.

Baseline ASPECTS was not significantly associated with cf DNA level ( $\mathrm{P}$ value=0.431). On further dichomitization between low (0-4) and interme-diate, ${ }^{5-7}$ ASPECT score was not significantly associated with cf DNA level. Higher ASPECTS combined with lower than 10,000 kilogenome-equivalent per liter cf DNA strongly predicted better neurological outcome at 90 days $(\mathrm{P}=0.003)$.

Mechanical thrombectomy or IVT was done in these 26 patients as per standard guidelines given by American Stroke Association. ${ }^{12}$ These were associated with improved outcome as measured by mRS three months score $(\mathrm{P}<0.05)$ (Fig. 1). Patients intervention by mechanical thrombectomy $(n=15)$ within six hours of presentation had significant impact on good outcome at three months $(\mathrm{P}<0.05)$ (Fig. 2).

The patients who underwent IVT and mechanical thrombectomy, or mechanical thrombec-tomy alone, achieved mTICI grade $2 \mathrm{~b}$ or $3^{13}$ in all cases. Further analysis at cf DNA level below 10,000 kilogenomeequivalent per liter showed better outcome at three months $(\mathrm{P}<0.05)$ (Table 2). Cut off of 10,000 kilogenome-equivalent per liter or more than three fold of normal value was based receiver operating curve analysis. The area under the curve was $0.79(95 \% \mathrm{CI}$, $0.67-0.92, \mathrm{P}=0.02)$ at $78 \%$ sensitivity and $83 \%$ specificity.

\section{DISCUSSION}

After initial clinical assessment, neuroimaging helps

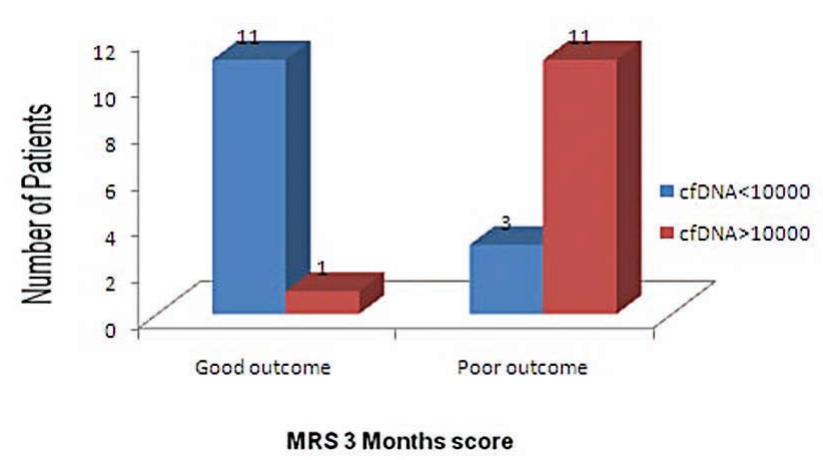

Fig. 1. Comparison of clinical outcome with 3-month mRS at cf DNA level of 10,000 in patients treated with therapeutic intervention combination of either mechanical thrombectomy or IVT or combined (P-value <0.05). to classify etiology as well as predict outcome in patients with acute ischemic stroke. However, clinical examination is subjective, and outcome prediction may be difficult at times. NCCT scan may not show the complete extent of damage in brain in acute settings, and few patients might be missed, despite of the robustness of the technology. ${ }^{14}$ Baseline infarction volume is an essential factor affecting clinical results in AIS along with the time-window. ${ }^{15}$ Many researchers have used ASPECTS to assess the efficiency of the treatment modality. This 10 -point quantitative topographic CT scan score can provide volumetric estimates of the size of cerebral infarction of anterior circulation. In IVT, the prognostic value of ASPECTS was established in clinical trials with large cohorts, showing a linear correlation between ASPECTS and prognosis. ${ }^{16}$ However, ASPECTS was not proven to modify the effectiveness of IVT in terms of improved functional outcomes in two randomized trials compared to placebo, ${ }^{17,18}$ and hence, does not support the exclusion of patients from IVT based on ASPECTS.

Unlike IVT, meta-analyses showed that high ASPECTS favored a good clinical outcome after endovascular therapy in comparison with low ASPECTS. ${ }^{19}$ However we did not find any significant association between ASPECT score and cf DNA level, probably because DNA release at cellular level may not be linearly reflected in early $\mathrm{CT}$ imaging. We performed baseline plain CT ASPECT scoring, not MRI ASPECT scoring. Although baseline ASPECTS and cf DNA were not significantly related, high $(\geq 8)$ ASPECT score combined with cf DNA less than 10,000 kilogenome-equivalent per liter showed better three months outcome (mRS 0-2) with a strong predictive value.

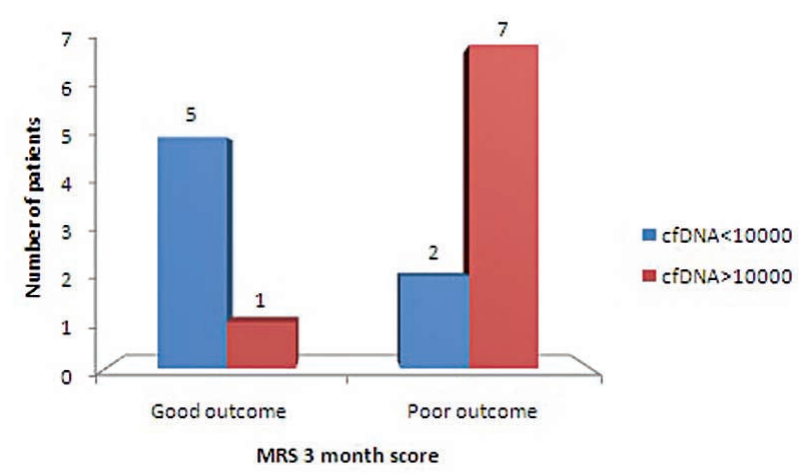

Fig. 2. Comparison of clinical outcome with 3-month $m R S$ at cf DNA level of 10,000 in patients treated with mechanical thrombectomy (P-value $<0.05)$. 


\section{Cf DNA- Outcome Prediction after Mechanical Thrombectomy in Stroke}

MRI brain is costly, and may not be available at all hospital set-ups. MRI is not suitable in some patients who are restless or patients with pacemakers or implants. Hence, we need to have a simple, cost effective, readily available biochemical marker for assessment of stroke severity and outcome.

Stroke is a complex disease with events like altered cerebral blood flow, ischemia to brain parenchyma, inflammation and neuronal damage that may result in apoptosis and neurologic dysfunction..$^{20}$ Increased $\mathrm{cf}$ DNA concentrations have been noted in conditions involving cell injury. ${ }^{21}$ Cellular ischemia and tissue infarction associated with stroke, as well as disruption of blood brain barrier, may lead to liberation of DNA from neuronal cells. Though the precise events of release of cf DNA in stroke are not clear, cell death or apoptosis is claimed to be a major factor. ${ }^{21,22}$ Enzymes like nucleases has been shown to degrade cf DNA present in peripheral blood. The half-life of cf DNA is about 10-15 minutes, due to rapid hepatic and renal clearance, ${ }^{3,14}$ and hence may be an ideal marker in stroke patients. Serial measurement of cf DNA may be more helpful for assessing ongoing brain damage due to short half-life. However, it is difficult to obtain precise optimal timing for blood collection within six hours frame due to differing time of patient arrival and difficulty in performing repeated sampling to arrive at precise optimal timing of sample collection.

Blood-based markers need to be identified to assess the severity of stroke for triage, optimal management, and prediction of treatment effect. The heterogeneity of stroke has hindered the establishment of specific blood biomarkers for assessing severity and prognosis. ${ }^{20}$ There is some evidence that HsCRP, a marker of inflammation, is also a marker of generalized atherosclerosis. Several studies have shown an association between elevated HsCRP concentrations and endothelial dysfunction in patients with coronary artery disease $^{23}$ but its role in stroke is not proven. We did not find any significant association between base-line blood sugar level, base-line HSCRP and stroke outcome. There is no definite evidence of blood biomarkers for assessing stroke outcome and even in the context of performing IVT or endovascular revascularization.

Although IVT remains the standard of care for acute ischemic stroke, patients with moderate to severe neurological deficits due to proximal artery occlusion have been found to improve with endovascular reperfusion therapy. ${ }^{24}$ With the advent of medical as well as surgical treatment options, there is a strong need to
Table 2. Significance of Cell Tree DNA at 10000 Levels with mRS at 3-Month Score

\begin{tabular}{lcc}
\hline Patient group & P-value & Odds Ratio at $95 \% \mathrm{Cl}$ \\
\hline MT & 0.020 & $17.50(3.61-450.21)$ \\
IVT & 0.012 & $21.00(1.22-350.35)$ \\
MT/IVT & 0.000 & $40.33(1.50-293.25)$ \\
\hline
\end{tabular}

establish better markers for predicting the effect of intervention. ${ }^{25} \mathrm{cf}$ DNA may be used as a marker for monitoring disease progress and outcome in stroke patients. Previous studies have reported-the correlations of cf DNA with infarct size, severity, morbidity and mortality in stroke patients. However, there is scarcity of data on cf DNA and stroke intervention..$^{11,14,22,25}$ This study intends to demonstrate the correlation of cf DNA concentration with stroke severity and outcome after intervention.

The strong correlation with mRS at 3 months score makes cf DNA suitable marker for prediction of stroke outcome (Table 2). The patients who underwent mechanical thrombectomy alone or either of the intervention (i.e., mechanical thrombectomy) or IVT had significantly better outcome when measured by 3 month $\mathrm{mRS}$. Thus, in patients with cf DNA level $>10,000$ intervention can be judiciously avoided (Table 2).

Thus, cf DNA can complement clinical examination and imaging tools to enhance the accuracy of stroke diagnosis. As a stroke biomarker, it can augment the diagnostic workup and help triage patients for intervention. With the addition of this novel marker, one may judiciously prognosticate about the outcome associated with mechanical thrombectomy or IVT in acute stroke patients. Considering the clinical outcome, efforts to hasten reperfusion therapy are always emphasized, regardless of the mode of intervention. The high costs associated with these interventions and the infrastructure that supports them needs to be balanced against the actual post intervention benefits. Along with clinical evaluation, cf DNA can be one such marker. It helps to determine the patients suitable for intervention. From this study, one can suggest to avoid costly treatment in patients less likely to improve, and therefore optimize the utility of resources in the stroke care unit.

Additional markers, like cf DNA, with prognostic utility can help patients and their kin to make informed decisions before giving consent for invasive or medical treatment. This may be especially helpful in cases 
where the imaging is negative or not indicated.

However, larger clinical trials with more elaborate study designs are suggested to establish the sensitivity and specificity of $\mathrm{cf}$ DNA for routine use in management of acute stroke. Since the success of stroke treatment requires rapid intervention, greater emphasis on improving rapid access and delivery of reperfusion therapies should be the priority.

\section{CONCLUSION}

Cf DNA concentration correlates well with the outcome of stroke intervention in acute ischemic stroke patients. Cf DNA as a prognostic surrogate marker to predict outcome in context with recanalization, post IVT and mechanical thrombectomy will help in optimal utilization of limited resources and lessen the burden on healthcare system. However, studies on larger sample size are suggested before extrapolating these findings.

\section{References}

1. The World Health Report: 2002: Reducing risks promoting healthy life. 2002. World Health Organization (WHO).

2. Jickling GC, Sharp FR; Blood Biomarkers of Ischemic Stroke, Neurotherapeutics 2011;8:349-360

3. Lo YMD, Tein MSC, Lau TK, Haines CJ, Leung TN, Poon PM, et al. Quantitative analysis of fetal DNA in maternal plasma and serum: implications for noninvasive prenatal diagnosis. Am J Hum Genet 1998;62:768-775

4. Lo YM, Rainer TH, Chan LY, Hjelm NM, Cocks RA. Plasma DNA as a prognostic marker in trauma patients. Clin Chem 2000;46:319-323

5. Bang OY, Goyal M, Liebeskind DS. Collateral Circulation in Ischemic Stroke: Assessment Tools and Therapeutic Strategies. Stroke 2015;46:3302-3309

6. Adams HPJr, Davis PH, Leira EC, Chang KC, Bendixen BH, Clarke WR, et al. Baseline NIH Stroke Scale score strongly predicts outcome after stroke: A report of the Trial of Org 10172 in Acute Stroke Treatment (TOAST). Neurology 1999;53;126-131

7. Banks JL, Marotta CA, Outcomes validity and reliability of the modified Rankin scale: implications for stroke clinical trials: a literature review and synthesis. Stroke 2007;38:1091-1096

8. National Institute of Neurological Disorders and Stroke rt-PA Stroke Study Group. Tissue plasminogen activator for acute ischemic stroke. N Engl J Med 1995;333:1581-1587

9. Saver JL, Jahan R, Levy EI, Jovin TG, Baxter B, Nogueira RG, et al. Solitaire flow restoration device versus the Merci retriever in patients with acute ischaemic stroke (SWIFT): a randomised, parallel-group, non-inferiority trial. Lancet 2012;380:1241-1249

10. Heid CA, Stevens J, Livak KJ, Williams PM. Real time quantitative PCR. Genome Res 1996;6:986-994
11. Rainer TH, Wong LKS, Lam W, Yuen E, Lam NY, Metreweli C, Lo YM, Prognostic use of circulating plasma nucleic acid concentrations in patients with acute stroke. Clin Chem 2003;49:562-569

12. Powers WJ, Derdeyn CP, Biller J, Coffey CS, Hoh BL, Jauch EC et al. American Heart Association/American Stroke Association focused update of the 2013 guidelines for the early management of patients with acute ischemic stroke regarding endovascular treatment: a guideline for healthcare professionals from the AHA/ASA. Stroke 2015;46:3020-3035

13. Zaidat OO, Yoo AJ, Khatri P, Tomsick TA, von Kummer R, Saver JL, et al, Recommendations on angiographic revascularization grading standards for acute ischemic stroke: a consensus statement. Stroke 2013;44:2650-2663

14. Lam NYL, Rainer TH, Wong KS, Lam W, Lo YM. Plasma DNA as a prognostic marker for stroke patients with negative neuroimaging within the first 24 hours of symptom onset. Resuscitation 2005;68:71-78

15. Schriger DL, Kalafut M, Starkman S, Krueger M, Saver JL. Cranial computed tomography interpretation in acute stroke: physician accuracy in determining eligibility for thrombolytic therapy. JAMA 1998;279:1293-1297

16. Hill MD, Buchan AM; Canadian Alteplase for Stroke Effectiveness Study I. Thrombolysis for acute ischemic stroke: results of the Canadian Alteplase for Stroke Effectiveness Study. CMAJ 2005;172:1307-1312

17. Dzialowski I, Hill MD, Coutts SB, Demchuk AM, Kent DM, Wunderlich O, et al. Extent of early ischemic changes on computed tomography (CT) before thrombolysis: prognostic value of the Alberta Stroke Program Early CT Score in ECASS II. Stroke 2006;37:973-978

18. Demchuk AM, Hill MD, Barber PA, Silver B, Patel SC, Levine $\mathrm{SR}$, et al. Importance of early ischemic computed tomography changes using ASPECTS in NINDS rtPA Stroke Study. Stroke 2005;36:2110-2115

19. Ryu CW, Shin HS, Park S, Suh SH, Koh JS, Choi HY. Alberta Stroke Program Early CT Score in the Prognostication after Endovascular Treatment for Ischemic Stroke: A Meta-analysis. Neurointervention 2017;12:20-30

20. Whiteley W, Chong WL, Sengupta A, Sandercock P. Blood markers for the prognosis of ischemic stroke: a systematic review. Stroke 2009;40:e380-e389

21. Fournie GJ, Martres F, Pourrat JP, Alary C, Rumeau M. Plasma DNA as cell death marker in elderly patients. Gerontology 1993; 39:215-221

22. Rainer TH, Wong KS, Lam W, Lam NY, Graham CA, Lo YM. Comparison of plasma $\beta$-globin DNA and S-100 protein concentrations in acute stroke, Clin Chim Acta 2007;376:190-196

23. Ridker PM, C-Reactive Protein: A Simple Test to Help Predict Risk of Heart Attack and Stroke, Circulation 2003;108:e81-e85

24. Prabhakaran S, Ruff I, Bernstein RA. Acute stroke intervention. A systematic review. JAMA 2015;313:1451-1462

25. Bustamante A, Mancha F, Macher HC, García-Berrocoso T, Giralt D, Ribó M, et al. Circulating cell-free DNA is a predictor of short-term neurological outcome in stroke patients treated with intravenous thrombolysis, J Circ Biomark 2016;5: 1849454416668791 


\section{COMMENTARY}

Jin Soo Lee, MD, PhD

Department of Neurology, Ajou University School of Medicine, Worldcup-ro 164, Yeongtong-gu, Suwon, Gyunggi-do, 16499, South Korea

Phone: +82.31 .219 .5175$

Fax: +82.31 .219 .5178$

E-mail: jinsoo22@gmail.com

Vajpeyee, et al. reported their study regarding cellfree DNA as a biomarker for predicting outcomes of mechanical thrombectomy in patients with acute ischemic stroke. ${ }^{1}$ The application of this new biomarker in the field of endovascular treatment is certainly interesting and worth some attention. Cell-free DNA has been recently developed as a novel biomarker in several diseases.

Despite their positive results, the current data need to be interpreted with caution. Cell-free DNA can be released from other comorbidities such as cancer and heart disease. ${ }^{2,3}$ However, comorbidities of patients were not analyzed or adjusted in the current study. There are limitations in this study and a larger prospective study is warranted. In future studies, sampling time should be strictly controlled, and infarct volume needs to be adjusted. In addition, The concept of cell-free DNA should be more clearly elucidated in other diseases because it still remains unclear. ${ }^{3}$ The role of cell-free DNA will then be further clarified in mechanical thrombectomy for those with acute ischemic stroke.

\section{References}

1. Vajpeyee A, Wijatmiko T, Vajpeyee M, Taywade O. Cell free DNA: A novel predictor of neurological outcome after IV thrombolysis and/or mechanical thrombectomy in acute ischemic stroke patients. Neurointervention 2018;13:13-18

2. Aarthy R, Mani S, Velusami S, Sundarsingh S, Rajkumar T. Role of Circulating Cell-Free DNA in Cancers. Mol Diagn Ther 2015;19:339-350

3. Lippi G, Sanchis-Gomar F, Cervellin G. Cell-free DNA for diagnosing myocardial infarction: not ready for prime time. Clin Chem Lab Med 2015;53:1895-1901 